1.35/1000 person-years. (see Ped Neur Briefs Jan 1995;9:1 for review and commentary). Cardiac causes for idiopathic seizures and a normal EEG, a possible explanation for some cases of SUD, especially in adolescent males, should always be considered when AEDs are ineffective.

\title{
INFANTILE SEIZURES
}

\section{INFANTILE SPASMS IN DOWN SYNDROME}

The clinical characteristics, EEG abnormalities, response to therapy, and outcome of 14 patients with infantile spasms and Down syndrome were studied at the Hopital Saint Vincent de Paul, Paris (9 cases); Universita Degli Studi de Pisa, Italy (2 cases); and Hopital de La Timone, Marseille, France (3 cases). None had antecedent cardiopathy or perinatal hypoxia. Spasms began between 4 and 18 months (mean 8 months), development was delayed before seizure onset, and visual contact deteriorated after seizure onset. Interictal EEGs showed typical hypsarrhythmia with no focal abnormality. Hydrocortisone (15 $\mathrm{mg} / \mathrm{kg} /$ day for 2 weeks, and discontinuation over 2 weeks) in 10 , and vigabatrin, valproate, or pyridoxine in 4 patients, controlled spasms and hypsarrhythmia within 6 months. Five with relapses within 2 months responded to further treatments. Seven remained seizure-free, and 7 developed other types of seizures resembling idiopathic generalized epilepsies, including myoclonic jerks, absences, or generalized atonic or tonic-clonic seizures, most responding readily to a combination of valproate, ethosuximide, and diazepam. None developed Lennox-Gastaut syndrome or other chronic refractory seizure disorder. Autistic features persisted in 2. (Silva ML, Cieuta C, Guerrini R, Plouin P, Livet MO, Dulac O. Early clinical and EEG features of infantile spasms in Down syndrome. Epilepsia Oct 1996;37:977-982). (Reprints: Dr O Dulac, Hopital Saint Vincent de Paul, 82 Ave Denfert Rochereau, 75674 Paris Cedex 14, France).

COMMENT. Infantile spasms in Down syndrome have the ictal and interictal EEG characteristics of idiopathic West syndrome, they respond relatively well to therapy, and do not generally evolve into Lennox-Gastaut or other chronic epilepsy syndrome. A delay in diagnosis may contribute to a worsening of cognitive dysfunction, and parents of Down syndrome children should be alerted to the possible development of spasms in the first year.

A case of West syndrome as the initial manifestation of congenital unilateral perisylvian cortical dysplasia is reported from University Children's Hospital, Badajoz; and Galicia General University Hospital, Santiago de Compostela, Spain (Vaquerizo-Madrid J, Eiris-Punal J, Gomez-Martin H et al. Acta Neuropediatr 1996;2:132-138). The child had left hemiatrophy and paresis and was developmentally delayed. Later, he had refractory epilepsy, with complex partial, atypical absence, and atonic seizures. The interictal EEG during sleep showed right sided epileptogenic activity with contralateral spread.

\section{PYRIDOXINE-DEPENDENT SEIZURES AND MRI ABNORMALITY}

An infant with pyridoxine-dependent seizures and MRI, PET, and EEG evidence of diffuse structural or functional brain disease is reported from the University of New Mexico Health Sciences Center, Albuquerque, NM, and the UCLA School of Medicine, Los Angeles, CA. Seizures began at 10 weeks, and status epilepticus occurred four times between 3 and 7 months of age. Trials of AEDs and ACTH were partially effective, but he became encephalopathic and 
hypotonic. EEG showed diffuse slowing with right posterior epileptiform discharges. A PET scan showed global cortical hypometabolism. Continued on phenobarbital monotherapy, he presented at 10 months with status and respiratory compromise. IV pyridoxine, $100 \mathrm{mg}$, controlled the seizure within 4 minutes, he was extubated after 1 day, and was maintained on $50 \mathrm{mg}$ pyridoxine daily. Phenobarbital was tapered without relapse. He gradually recovered muscle tone, walked at 15 months, and was seizure-free at 20 months, but speech was delayed. MRI showed diffuse cortical atrophy, especially frontal. (Shih JJ, Kornblum H, Shewmon DA. Global brain dysfunction in an infant with pyridoxine dependency: evaluation with EEG, evoked potentials, MRI, and PET. Neurology Sept 1996;47:824-826). (Reprints: Dr JJ Shih, Neurology Department, University of New Mexico Health Sciences Center, 915 Camino de Salud, NE, Albuquerque, NM 87131).

COMMENT. Pyridoxine-dependent seizures may be complicated by structural and functional brain disease. These abnormalities demonstrated by MRI and PET may result from the metabolic dysfunction secondary to pyridoxine-dependency, but the effects of hypoxia with repeated episodes of status epilepticus and possibly the use of ACTH in treatment of the seizures could have contributed to the cerebral atrophy demonstrated by MRI. The remarkable clinical recovery of this patient, after the diagnosis was made and specific treatment initiated at 10 months, emphasizes the importance of a trial of pyridoxine for intractable epilepsy, even in older infants and children.

\section{HEADACHE DISORDERS}

\section{PREVALENCE OF HEADACHE IN SCHOOL CHILDREN}

The prevalence of migraine and other headaches in 7-year-old children, in 1974 and 1992, was determined by school physicians at the time of medical examinations, using the identical study design and a similar urban child population of the same age group, and the data analysed in the Department of Child Neurology, University of Turku, Finland. The prevalence of "present" headache at age 7 years, defined as headache occurring in the preceding 6 months, increased from $14.4 \%$ in 1974 to $51.5 \%$ in 1992 . Boys and girls were affected similarly. "Past" headache, having occurred at any time prior to present headache, also showed a significantly increased prevalence from $23.4 \%$ of children affected in the 1974 study to $71.1 \%$ in 1992 . In both present and past headache categories, those having headaches infrequently. less than once a month or yearly, showed the greatest increase in prevalence. Precipitating factors, fever, fatigue, and head trauma, were equally prevalent in 1974 and 1992. Migraine headache had also increased from 1.9\% affected in 1974 to $5.7 \%$ in 1992 . Migraine prevalence was especially high in city areas with high percentages of council houses and family relocations. (Sillanpaa M, Anttila P. Increasing prevalence of headache in 7-year-old schoolchildren. Headache Sept 1996;36:466-470). (Respond: Dr Matti Sillanpaa, University of Turku Hospital, TYKS, 20520 Turku, Finland).

COMMENT. The prevalence of headache, including migraine, has increased significantly in school age children living in an urban area in Finland, in an 18 year period from 1974 to 1992 . Highest increases occur among children exposed to social instability and stress. Similar increases in headache prevalence are known to have occurred in adults in the United States in the 1980s. (see Progress in Pediatric Neurology II, PNB Publ, 1994, pp153-155). 